\title{
Understanding Etiology of Complex Neurodevelopmental Disorders: Two Approaches
}

\author{
Andrey Rzhetsky \\ Depts. of Medicine and Human Genetics, Computation Institute and Institute of Genomics and \\ Systems Biology, University of Chicago, Chicago, Illinois, USA \\ arzhetsk@medicine.bsd.uchicago.edu
}

\begin{abstract}
Complex human phenotypes, such as autism, schizophrenia, and anxiety, undoubtedly partially overlap in genomic variations that predispose to or protect against these maladies. (Genetic overlap of complex phenotypes has gained increasing experimental support and is no longer just an ungrounded scientific hypothesis.) Furthermore, as yet largely unknown shared environmental factors likely tend to trigger the manifestation of more than one phenotype. Although it may seem overly ambitious to target multiple phenotypes jointly, we believe we can obtain much more information from existing data and gain new insights into individual phenotypes by modeling phenotypes jointly. My talk sketches two distinct computational approaches to this problem.
\end{abstract}

Keywords: computational approaches for modeling joint phenotypes, neurodevelopmental disorders. 